\title{
Brief ideas about evidence-based recovery in team sports
}

\author{
Julio Calleja-González ${ }^{1 * *}$, Juan Mielgo-Ayuso ${ }^{2}$, Jaime Sampaio ${ }^{3}$, Anne Delextrat ${ }^{4}$, Sergej M. Ostojic ${ }^{5}$, Diego Marques-Jiménez', \\ Iñaki Arratibel', Braulio Sánchez-Ureña ${ }^{6}$, Gregory Dupont ${ }^{7}, X^{1}$ avi Schelling ${ }^{8}$, Nicolás Terrados ${ }^{9}$ \\ 'Department of Physical Activity and Sports, University of the Basque Country, Vitoria, Spain \\ 2European University of Madrid, Madrid, Spain \\ ${ }^{3}$ Research Center in Sports Sciences, Health Sciences and Human Development (CIDESD), CreativeLab Research Community, Vila Real, Portugal \\ ${ }^{4}$ Sport and Health Sciences Department, Oxford Brookes University, Oxford, UK \\ ${ }^{5}$ University of Belgrade School of Medicine, Belgrade, Serbia \\ ${ }^{6}$ Human Movement and Quality Life School, Universidad Nacional de Costa Rica, Heredia, Costa Rica \\ 'Université de Lille, EA 7369 - URePSSS - Unité de Recherche Pluridisciplinaires Sport Santé Société, Research Department, Lille Olympique Sporting Club (LOSC), Lille, France \\ ${ }^{8}$ Complex Systems in Sport Research Group SGR, National Institute of Physical Education of Catalonia (INEFC), University of Lleida, Lleida, Spain \\ ${ }^{9}$ Department of Functional Biology, University of Oviedo, Oviedo, Spain
}

Performance in team sports is the expression of complex, dynamic, interactive, and multidimensional processes. It is now well-established that optimum recovery after practice or match is a key factor of team sport performance. During season and tournaments, improving recovery could offer an advantage for following performance. As a consequence of the professionalization of different roles in staffs, new particular roles have been developed within the team sports physician core in order to improve recovery protocols. Presently, scientific literature presents a big amount of methods used to enhance recovery based on the type of practice, time between session or competitions and equipment and/or staff accessible. These practices, usually used by teams are related to: ergonutritional, water therapy, massages techniques, stretching compression garments, sleep strategies and psychological implements. Besides, travel fatigue has been recognized by athletes and coaches as a challenging problem that could benefit from practical solutions. Nowadays, players have to play a lot of matches without enough time to recover among them, therefore the use of well-managed recovery can lead to a competitive advantage. Although the main purpose of applied sport sciences investigation is to categorize the protocols as well as providing approaches for individual recovery, the stages to recognize the most appropriate recovery plans in the field of team sports come from the analysis of the individual parameters.

Keywords: Recovery, Performance, Team sports

\section{INTRODUCTION}

Performance in team sports is the expression of complex and dynamic multidimensional and interactive processes. In fact, preparing players to respond to actual game demands has led to fine-tuning several important recovery-related topics, consensually considered to enhance performance, such as: (a) Injury prevention programmes and practice processes to optimize return to training and return to play (Carling et al., 2016), (b) Management of high intensity training using optimized and individualized methods: repeated sprint ability and similar approaches, small-sided games or high-intensity interval training (Bangsbo, 2015), (c) Personalized and individual recovery for practice and competition (Calleja-González et al., 2016) based on individual fatigue and minimization of the effects of travel fatigue (Benjamin et al., 2015). Travelling long distances by plane presents a large effect on subjective grades of jet-lag, neurological fatigue and sleepiness. In particular, sleep and subjective responses are conceded, reflecting the travel requirements, matches atmosphere and environmental conditions (Thornton et al., 2018) and (d) Adequate testing process of

\footnotetext{
*Corresponding author: Julio Calleja-González

(iD https://orcid.org/0000-0003-2575-7168

Department of Physical Education and Sport, Faculty of Physical Activity and Sport Sciences, University of the Basque Country (UPV-EHU), Portal de Lasarte, 71, 1007 Vitoria-Gasteiz, Spain

Tel: +34-945-013-525, Fax: +34-945-013-501

E-mail: julio.calleja.gonzalez@gmail.com

Received: May 2, 2018 / Accepted: June 25, 2018
} 


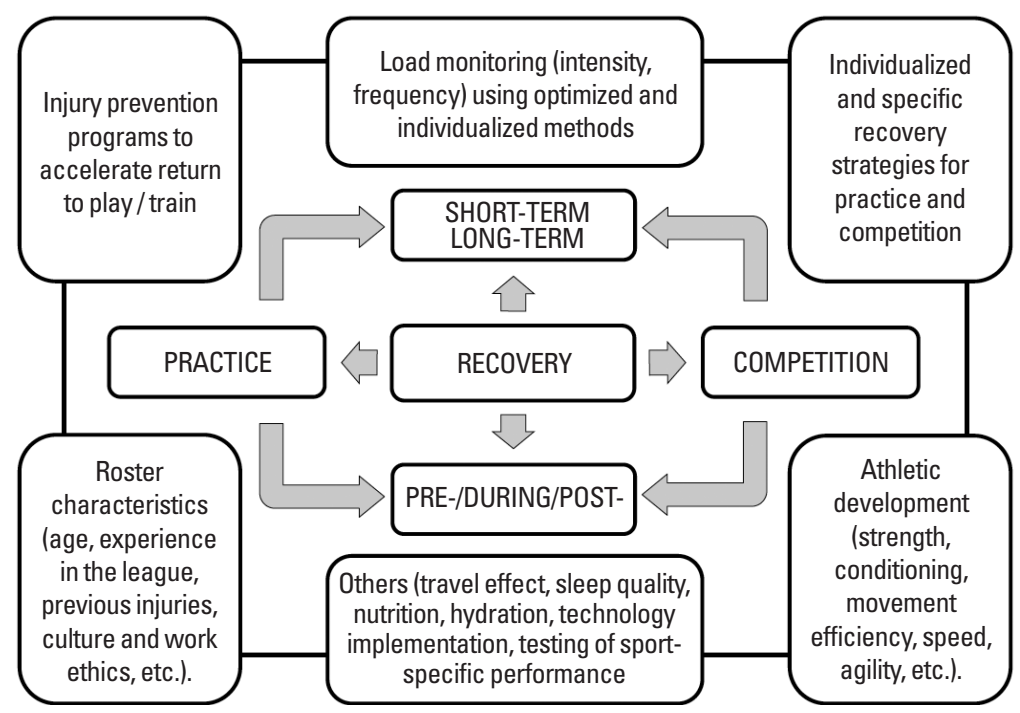

Fig. 1. Framework of recovery.

sport-specific performance with an appropriate use of monitoring technology (Coutts, 2014), under implementation of technology in modern sports teams (Torres-Ronda and Schelling, 2017). Moreover, main concrete specific mechanisms of fatigue with ad hoc training load analysis could inform about the most appropriate recovery methods (Halson, 2014). Within this proposed framework (Fig. 1), the current manuscript aims to describe brief insights about current evidence-based recovery in team sports.

\section{THE CONCEPT OF RECOVERY}

It is now well-established that optimal recovery from practice or matches plays a major role on team sport performance. During competitive periods and tournaments, where players may compete numerous times over a few days, enhancing recovery may provide a decisive advantage for subsequent performance. Top level players dedicate a much higher proportion of their daily time in recovery than they do in practice (Terrados et al., 2009). Besides, some authority authors in this field concluded that the "recovery concept" needs more research. Nonetheless, an effort to increase the transfer of training to sport performance, sport-specific training programs should be developed. The competition modelling has been suggested as a new method for developing metabolic conditioning programs similar to matches (Rhea et al., 2006), for this reason new recovery ideas are in the scientific community (Ostojic, 2016). Precisely defining "recovery from exercise" is a challenging mission due to the mix concepts of recovery. This pioneer idea inspires a multifactorial approach to the "physiology of recovery" and evidences more research on the near future (Ostojic, 2016). One of the most interesting changes involves the nervous system and its influences on the type of exercise performed (Taylor et al., 2016).

\section{APPLIED SPORT SCIENCE KNOWLEDGE FOR RECOVERY}

The guidelines for team physician's staff provide evidence-based information as a result of the specialization of different roles in team sports work and based on the last Position Stand "Team Physician Consensus Statement, 2013" (Herring et al., 2013). In the last years, new specific roles have been involved within team sports staff in order to improve recovery routines. Amongst them, the "applied sport scientist focus on recovery" is presented as an alternative in teams and clubs, intending to establish the complex process of recovery of players.

\section{FATIGUE DIAGNOSIS}

The compression of type of fatigue in different team sports and appropriate monitoring of training load may offer relevant data about practices and adequate recovery methods (Halson, 2014). Moreover, it is vital to preserve players' health and the most common illnesses in these types of athletes, in particular previous injuries (Brockett et al., 2004). On the other hand, tactical systems seem to be affected by fixtures distribution or noncongested fixtures (Folgado et al., 2015), given that players cover total distanc- 
es at similar intensities in both situations. For that, the reduction of synchronization could be related to an increased perception of fatigue (Folgado et al., 2015). Many variables have been described in the scientific literature (Halson, 2014) to understand the training load and the potential effect on players. Among them, heart rate variability and heart rate recovery are the two of the most common to understand training adaptation. As well as other physiological parameters (blood, urinary and hormonal variations) (Djaoui et al., 2017), and rate perception scales (Fox et al., 2017), etc.

As long as analyses may be completed regularly and rapidly enough and with minimal interference with practice or competition, it will be possible to apply ad hoc individualized recovery protocols. Within this context, sports technicians have a big variety of markers in order to monitoring the fatigue (Wiewelhove et al., 2015), as well as for example, novel methodology to measure skeletal muscle glycogen content through rapid, noninvasive high-frequency ultrasound (Hill and Millán, 2014), given that ingesting carbohydrate immediately after training and competition (metabolic window) will rapidly recover liver and muscle glycogen pool (Williams and Rollo, 2015). Finally, in the last 2 years other interesting topic has come up to scene (acute:chronic workload ratio) as predictor of load-related injuries (Gabbett, 2016).

\section{ORGANIZATION OF THE PROCESS}

Now at days the recovery methods have been defined based on type of activity, time between training sessions or competitions, and the medical staff availability (Calleja-González et al., 2016), although scarce literature with a high level of evidence in team sports has been published. Finally, to mention appropriate protocols to enhance recovery, the scientific way try to provide a greater understanding of the physiology underpinning performance change, recovery approaches and important factors to contribute to the diverse physiological responses in players (Stephens et al., 2017).

\section{PROTOCOLS WITH HIGH LEVEL OF EVIDENCE AND HIGH GRADES OF RECOMMENDATION}

These practices usually used by teams are related to: ergonutritional, water therapy, massages techniques, stretching compression garments, sleep strategies and psychological implements (Calleja-González et al., 2016). However, there is an absence of agreement on the benefits of some of these methods in the scientific literature (Calleja-González et al., 2016) and on their best in- fluence: in pre, per, and postpractice/competition. In our case, we suggest this classification of recovery methods (Vaile et al., 2010). Presently, the scientific literature in the area of recovery was examined to collate evidence regarding the effectiveness of popular recovery interventions. Therefore, to quantify their effectiveness, each recovery strategy or subcategory was given a rating of the misreading of recommendation as relating to its importance, rather than to the strength of the supporting evidence, and may therefore fail to give due weight to low grade recommendations (Australian Institute of Sport, 2014).

\section{MANAGEMENT STRATEGIES TO REDUCE TRAVEL FATIGUE}

Long-distance trips are becoming common in professional team sports (Steenland and Deddens, 1997). Any long journey by: plane, train or road is related with "travel fatigue," given that the combined effects of a changed routine (sleep loss or altered meals) and the general disruption caused by travel (Reilly et al., 2008). The influence of transcontinental travel and high-volume travel on team sports players induce in physiological disorders and complex physical symptoms (Kraemer et al., 2016). The most relevant are: fatigue, reduced alertness, loss of appetite and disturbance of the sleep cycle (Srinivasan et al., 2010). It is suggested that this latter phenomenon is at least partly due to a lack of adequate training time during the period of long-distance travel and competing. Long time ago travel fatigue has been known by sports community as inspiring problems that could benefit from practical resolutions (Hanson, 1975; Samuels, 2012). Amongst them, peripheral oedema has been described (Clarke et al., 2016), as the continued sitting in plane seats is related with a fluid accumulation in the legs during the first hours (Mittermayr et al., 2007). Therefore, low-ankle-pressure graduated-compression tights may reduce flight-induced ankle oedema and subjectively-rated symptoms (Hagan and Lambert, 2008). In this way, some methods are designated to reduce neuromuscular fatigue in tournaments after long-haul travel (Mitchell et al., 2017).

\section{RECOVERY UNITS}

Now at days team sports participants compete many times without enough recovery periods (Calleja-González et al., 2016), thus the use of well-managed recovery can lead to a competitive advantage (Terrados et al., 2009). To address the demands of competition, the reference countries in Sport Sciences (e.g., Australian 
proposal during Olympics), implemented recovery mobile units, moving its departmental structure from the Australian Institute of Sport to the location of competitions. It includes a physical space for the team, where players can go after their workouts or competitions, to benefit from a specific recovery protocol according to their primary energy pathways or their type of training (strength/speed/power or intermittent or endurance).

\section{THE FUTURE OF RECOVERY IN TEAM SPORTS...?}

Among others, identifying which recovery methods in the field of sports sciences offer solutions for individual recovery (Moreno et al., 2015) come from the analysis of the following individual parameters:

\section{Quantification of training load, player's responses before starting practice or competition (sleepiness, recovery profile, stress, muscle pain, and fatigue)}

These ideas describe the hypothesis that in periods of high loads (a lot of matches in brief periods of time), there is a risk that players could overestimate the intensity of practice done. New conception about it, describes 'over-estimators' or 'subestimators' of training loads (Borresen and Lambert, 2008). This is the main reason that we must be cautious when making evaluations among players. If a player describes a higher load versus other player, it does not mean that he has accumulated a greater load, but it may be an overestimator.

\section{Responders or nonresponders}

The response to an exercise programs is habitually defined with the assumption that the average group typifies a characteristic response. Currently, we can observe a wide range of responses to a same intervention among different players. Based on concept of 'high responders' or 'low responders' after a standardized practice intervention this could offer us interesting information about individual adaptations to practice or competition (Mann et al., 2014).

\section{Intraindividual variability}

This modern theory elucidates to the oscillation of biological factors in the same individual considering their personal conditions which could be identified or not. In this way, the biological function, does not imply that it has to necessarily produce other changes in different organic systems that have different biological organic rhythms. To support this idea, studies form the last years presented the recollection of different parameters (genes, haematological parameters) offer concrete information about the relation between intraindividual and inter-individual variability (Saugy et al., 2014).

Related to thus new topic, a referent author in the field of sport sciences describers the new role of epigenetics, understanding the role of genomic variation in human performance. The interaction with genomics and transcriptomics by bioinformatics tools and precise phenotyping may detail classification of the test populations. These ideas will present better knowledge about the interrelations between exercise physiology and performance. The combination input could explain the outcome to understand the molecular basis of physical performance in humans (Ehlert et al., 2013).

\section{THE PHILOSOPHY OF WORK}

To conclude, as part of the work philosophy of sport scientists focusing on recovery, we can present some strategies for action as part of our team's work, creating an interaction among specialists to highlight strengths that can optimize the care and treatment of team sport players (Chung et al., 2017). On the other hand, the ethics of this proposal align well with certain moral goals, like treating the player's interests more fairly and treating the player's health as an end instead of as the means to an end. The methods and tools do not always analyse the cost-benefit of the implementations (Buchheit, 2014; McKinney, 2016; Torres-Ronda and Schelling, 2017). Therefore we propose simple interventions (Hill and Millán, 2014), no interference with the rest of the staff (specially with the Head coach), not confrontation with players, nonimposition yes-conviction, invest more time to inform the player and explain the details (final report for player) and finally to provide an individualistic approach to evaluating and monitoring recovery to attend more accurately to each player's recovery needs (Moreno et al., 2015).

\section{CONCLUSIONS}

Recovery from exercise is part of team sports performance during density periods of competition $s$ and tournaments, where players compete many times in a brief period of time. In addition, the use of recovery strategies is a relatively new area in the sport sciences field. As a result of the specialization in team sports work, new specific roles have been included within team sports physi- 
cian staff in order to improve recovery routines. Understanding specific mechanisms of fatigue in team sports and appropriate monitoring of training load can provide important information about training and recovery strategies. For that, many recovery protocols presented to enhance recovery processes have been recommended based on model of practice, rest the time between practice and matches or equipment facilities. Therefore, to quantify their effectiveness, each recovery strategy or subcategory was based on the current scientific knowledge. Finally, the steps to identify the best recovery strategies in the field of team sports come from the analysis of the following individual parameters and interactions among coaches, physicians and medical specialists to highlight strengths that can optimize the care and treatment of sports team players, and the combination of different strategies based on the type of fatigue.

\section{CONFLICT OF INTEREST}

No potential conflict of interest relevant to this article was reported.

\section{REFERENCES}

Australian Institute of Sport [Internet]. Bruce (Australia): Australian Institute of Sport; 2014 [cited 2018 Mar 23]. Adapted from: http://www. ausport.gov.au/ais.

Bangsbo J. Performance in sports: with specific emphasis on the effect of intensified training. Scand J Med Sci Sports 2015;25 Suppl 4:88-99.

Benjamin HJ, Boniquit NT, Hastings ES. The traveling athlete. Adolesc Med State Art Rev 2015;26:189-207.

Borresen J, Lambert MI. Autonomic control of heart rate during and after exercise: measurements and implications for monitoring training status. Sports Med 2008;38:633-646.

Brockett CL, Morgan DL, Proske U. Predicting hamstring strain injury in elite athletes. Med Sci Sports Exerc 2004;36:379-387.

Buchheit M. Monitoring training status with HR measures: do all roads lead to Rome? Front Physiol 2014;5:73.

Calleja-González J, Terrados N, Mielgo-Ayuso J, Delextrat A, Jukic I, Vaquera A, Torres L, Schelling X, Stojanovic M, Ostojic SM. Evidencebased post-exercise recovery strategies in basketball. Phys Sportsmed 2016;44:74-78.

Carling C, McCall A, Le Gall F, Dupont G. The impact of short periods of match congestion on injury risk and patterns in an elite football club. Br J Sports Med 2016;50:764-768.

Chung KC, Lark ME, Cederna PS. Treating the Football athlete: coaches' perspective from the University of Michigan. Hand Clin 2017;33:1-8.

Clarke MJ, Broderick C, Hopewell S, Juszczak E, Eisinga A. Compression stockings for preventing deep vein thrombosis in airline passengers. Cochrane Database Syst Rev 2016;9:CD004002.

Coutts AJ. In the age of technology, Occam's razor still applies. Int J Sports Physiol Perform 2014;9:741.

Djaoui L, Haddad M, Chamari K, Dellal A. Monitoring training load and fatigue in soccer players with physiological markers. Physiol Behav 2017;181:86-94.

Ehlert T, Simon P, Moser DA. Epigenetics in sports. Sports Med 2013;43: 93-110.

Folgado H, Duarte R, Marques P, Sampaio J. The effects of congested fixtures period on tactical and physical performance in elite football. J Sports Sci 2015;33:1238-1247.

Fox JL, Scanlan AT, Stanton R. A review of player monitoring approaches in basketball: current trends and future directions. J Strength Cond Res 2017;31:2021-2029.

Gabbett TJ. The training-injury prevention paradox: should athletes be training smarter and harder? Br J Sports Med 2016;50:273-280.

Hagan MJ, Lambert SM. A randomised crossover study of low-anklepressure graduated-compression tights in reducing flight-induced ankle oedema. Med J Aust 2008;188:81-84.

Halson SL. Monitoring training load to understand fatigue in athletes. Sports Med 2014;44 Suppl 2:S139-147.

Hanson JS. Decline of physiologic training effects during the competitive season in members of the U.S. Nordic ski team. Med Sci Sports 1975;7: 213-216.

Herring SA, Kibler WB, Putukian M. Team physician consensus statement: 2013 update. Med Sci Sports Exerc 2013;45:1618-1622.

Hill JC, Millán IS. Validation of musculoskeletal ultrasound to assess and quantify muscle glycogen content. A novel approach. Phys Sportsmed 2014;42:45-52.

Kraemer WJ, Hooper DR, Kupchak BR, Saenz C, Brown LE, Vingren JL, Luk HY, DuPont WH, Szivak TK, Flanagan SD, Caldwell LK, Eklund D, Lee EC, Häkkinen K, Volek JS, Fleck SJ, Maresh CM. The effects of a roundtrip trans-American jet travel on physiological stress, neuromuscular performance, and recovery. J Appl Physiol (1985) 2016;121: 438-448.

Mann TN, Lamberts RP, Lambert MI. High responders and low responders: factors associated with individual variation in response to standardized training. Sports Med 2014;44:1113-1124

McKinney R Jr. Being right isn't always enough: NFL culture and team physicians' conflict of interest. Hastings Cent Rep 2016;46 Suppl 2:S3334.

Mitchell JA, Pumpa KL, Pyne DB. Responses of lower-body power and 
match running demands following long-haul travel in international rugby sevens players. J Strength Cond Res 2017;31:686-695.

Mittermayr M, Fries D, Gruber H, Peer S, Klingler A, Fischbach U, Gunga

HC, Koralewski E, Faulhaber M, Simmer M, Schobersberger W. Leg edema formation and venous blood flow velocity during a simulated long-haul flight. Thromb Res 2007;120:497-504.

Moreno J, Ramos-Castro J, Rodas G, Tarragó JR, Capdevila L. Individual recovery profiles in basketball players. Span J Psychol 2015;18:E24.

Ostojic SM. Editorial: post-exercise recovery: fundamental and interventional physiology. Front Physiol 2016;7:3.

Reilly T, Waterhouse J, Edwards B. A review on some of the problems associated with long-distance journeys. Clin Ter 2008;159:117-127.

Rhea MR, Hunter RL, Hunter TJ. Competition modeling of American football: observational data and implications for high school, collegiate, and professional player conditioning. J Strength Cond Res 2006;20:5861.

Samuels $\mathrm{CH}$. Jet lag and travel fatigue: a comprehensive management plan for sport medicine physicians and high-performance support teams. Clin J Sport Med 2012;22:268-273.

Saugy M, Lundby C, Robinson N. Monitoring of biological markers indicative of doping: the athlete biological passport. Br J Sports Med 2014;48:827-832.

Srinivasan V, Singh J, Pandi-Perumal SR, Brown GM, Spence DW, Cardinali DP. Jet lag, circadian rhythm sleep disturbances, and depression: the role of melatonin and its analogs. Adv Ther 2010;27:796-813.
Steenland K, Deddens JA. Effect of travel and rest on performance of professional basketball players. Sleep 1997;20:366-369.

Stephens JM, Halson S, Miller J, Slater GJ, Askew CD. Cold-water immersion for athletic recovery: one size does not fit all. Int J Sports Physiol Perform 2017;12:2-9.

Taylor JL, Amann M, Duchateau J, Meeusen R, Rice CL. Neural contributions to muscle fatigue: from the brain to the muscle and back again. Med Sci Sports Exerc 2016;48:2294-2306.

Terrados N, Calleja-González J, Jukic I, Ostojic SM. Physiological and medical strategies in post-competition recovery-practical implications based on scientific evidence. Ser J Sports Sci 2009;3:29-37.

Thornton HR, Miller J, Taylor L, Sargent C, Lastella M, Fowler PM. Impact of short- compared to long-haul international travel on the sleep and wellbeing of national wheelchair basketball athletes. J Sports Sci 2018;36:1476-1484.

Torres-Ronda L, Schelling X. Critical process for the implementation of technology in sport organizations. Strength Cond J 2017;39:54-59.

Vaile J, Halson S, Graham S. Recovery review: science vs. practice. J Aust Strength Cond 2010;18(Suppl 2):5-21.

Wiewelhove T, Raeder C, Meyer T, Kellmann M, Pfeiffer M, Ferrauti A. Markers for routine assessment of fatigue and recovery in male and female team sport athletes during high-intensity interval training. PLoS One 2015;10:e0139801.

Williams C, Rollo I. Carbohydrate nutrition and team sport performance. Sports Med 2015;45 Suppl 1:S13-22. 